\title{
Theoretical analysis of the adsorption of Ammonia-Borane and their dehydrogenation products on the (001) surface of TiC and ZrC
}

\author{
${ }^{\mathrm{a}, \mathrm{b}}$ Andrea Echeverri, ${ }^{\mathrm{b}, \mathrm{c}}$ Carlos Cárdenas, ${ }^{\mathrm{d}}$ Monica Calatayud, ${ }^{\mathrm{a} C a c i e r}$ Zilahy Hadad, ${ }^{\mathrm{e}}$ Tatiana Gomez \\ ${ }^{a}$ Grupo de Química-Física Teórica, Instituto de Química, Universidad de Antioquia, Calle 70 No. 52- \\ 21, Medellín, Colombia \\ ${ }^{b}$ Departamento de Física, Facultad de Ciencias, Universidad de Chile, 653-Santiago, Chile \\ 'Centro para el desarrollo de la Nanociencias y Nanotecnología, CEDENNA, Av. Ecuador 3493 \\ Santiago, Chile \\ ${ }^{d}$ Sorbonne Universités, UPMC Univ Paris 06, CNRS, Laboratoire de Chimie Théorique, CC 137-4, \\ Place Jussieu F, 75252 Paris Cedex 05, France \\ ${ }^{\mathrm{e}}$ Theoretical and Computational Chemistry Center, Institute of Applied Chemical Sciences, Faculty of \\ Engineering, Universidad Autonoma de Chile, El Llano Subercaceaux 2801, Santiago, Chile
}

\section{ABSTRACT}

The adsorption of ammonia-borane (AB) over the (001) surface of TiC and $\mathrm{ZrC}$, has been studied systematically by means of periodic-boundary density functional calculations using slab models. We present evidence that a surface with an appreciable degree of polarity such as those used in this research, can simultaneously activate the $\mathrm{N}-\mathrm{H}$ and $\mathrm{B}-\mathrm{H}$ bonds of the $\mathrm{AB}$ and its dimer $\mathrm{AB}-\mathrm{AB}$. The molecule is highly activated by both supports, and the $\mathrm{N}-\mathrm{H}$ and $\mathrm{B}-\mathrm{H}$ bonds are stretched long enough to release one or two hydrogens. Additionally, the bond distance $\mathrm{B}-\mathrm{N}$ is shortened by 0.04 and $0.06 \AA$ by TiC and ZrC supports respectively, which results in the strengthening of the bond. A systematic study for the adsorption of $\mathrm{BH}_{2} \mathrm{NH}_{2}$, which is isoelectronic with ethylene, was also done. The adsorption leads to a major elongation of the $\mathrm{B}-\mathrm{N}$ bond with respect to the calculated value in the gas phase $(0.17$ and $0.18 \AA$ for $\mathrm{TiC}$ and $\mathrm{ZrC}$ respectively). Both supports can activate the molecule, with the biggest impact being on the $\mathrm{BH}_{2}$ fragment. On the other hand, the migration of hydrogen atoms on both supports is a thermodynamically favorable process. On the surface $\mathrm{H}$ prefers bonding to $\mathrm{a} C$, which is in agreement with other studies for dissociative adsorption of $\mathrm{H}_{2}$ on metallic carbides. Nevertheless, we show that the hydrogen molecule readily forms when both atoms sit on the transition metal of the surface. 


\section{Introduction}

Hydrogen-based energy has been studied and highlighted as an alternative source of fossil fuels, given that the combustion of hydrogen is approximately three times more energetic than gasoline, and its only waste is water, which makes it friendly to the environment. ${ }^{1}$ Additionally, the availability of molecular hydrogen as a reactant is highly relevant in catalytic processes related to the chemical industry, such as the reduction and hydrogenation of compounds, and the refinement of petroleum. ${ }^{2}$ In many of these industrial processes the presence of activated hydrogen in atomic form is necessary, which is achieved by using various catalysts. ${ }^{3-9}$ Among lightweight hydrogen storage materials, ammonia-borane $\left(\mathrm{AB}, \mathrm{BH}_{3} \cdot \mathrm{NH}_{3}\right)$ has received much attention in recent years because of its high gravimetric $\left(19.6 \mathrm{wt} \% \mathrm{H}_{2}\right)$ and volumetric hydrogen densities, and it has thus been explored extensively as a potential candidate for chemical hydrogen storage. ${ }^{10,11}$ This substance is stable in the solid state due to the strong dihydrogen interactions of the type $\mathrm{NH}-\mathrm{HB}$ (the dissociation of $\mathrm{H}-\mathrm{H}$ interactions requires $90 \mathrm{kcal} / \mathrm{mol}$ ), and it contains a greater amount of hydrogen per unit volume than liquid hydrogen. ${ }^{10}$ It is soluble in methanol, water, and ether, among other polar solvents. ${ }^{10}$ The use of solvents alone, while accompanied by transition metals in the form of nanoparticles or nanocomposites, has been particularly promising for dehydrogenation. ${ }^{12-16}$ Studies in this regard have focused on exploring various crystalline catalysts, ${ }^{17-19}$ amorphous, $^{13}$ alloys, ${ }^{13}$ nanoparticles, ${ }^{12-16,20-22}$ and core-shell ${ }^{13,20-22}$ structures, with results varying from the amount of hydrogen that it can be released in catalysis, to time and waste generated in the reaction. Satisfactory results are obtained at low temperatures or at room temperature with metals like platinum, ${ }^{12,17}$ cobalt, ${ }^{13,14,18,23-25}$ nickel, ${ }^{15,16,18}$ rhodium, ${ }^{12}$ ruthenium, ${ }^{12,26-}$ 30 and copper. ${ }^{18}$ In addition, different supports have been explored for these nanoparticles, including $\mathrm{SiO}_{2}{ }^{12,18} \mathrm{Y}_{-} \mathrm{Al}_{2} \mathrm{O}_{3}{ }^{12,18}$ and carbon. ${ }^{12,15,20}$ However, the use of $\mathrm{AB}$ as a material for hydrogen storage leads us to understand some disadvantages, such as: its low release efficiency, high working temperatures, and unwanted by-products $\left(\mathrm{NH}_{3}, \mathrm{BH}_{3}\right.$, etc $) \cdot{ }^{18,31-40}$ In this sense, there is a continuous search for new hydrogenation catalysts and materials that can adsorb and dissociate $\mathrm{H}_{2}$ in an efficient way. ${ }^{41-43}$

Transition metal carbides (TMC) exhibit physical and chemical properties similar to that of very expensive noble metals ( $\mathrm{Pt}, \mathrm{Pd}, \mathrm{Ru}$, or $\mathrm{Rh}),{ }^{44}$ and they have been used to improve the catalytic activity of important industrial processes such as: hydrogenation reactions, ${ }^{45,46}$ dehydrogenation, ${ }^{47,48}$ isomerization, ${ }^{49}$ and especially, in desulphurization reactions, ${ }^{45,50,51}$ thanks to 
their high resistance to sulfur poisoning. Metal clusters supported on TMC have been used in the breakdown of the $\mathrm{H}-\mathrm{H}$ bond of $\mathrm{H}_{2}$, where the adsorption and dissociation of $\mathrm{H}_{2}$ has been evaluated. ${ }^{52-54}$ The results show that small Au clusters in contact with $\mathrm{TiC}(001)$ exhibit chemical properties not seen in the gas phase or Au supported on oxide surfaces, making $\mathrm{Au} / \mathrm{TiC}(001)$ an excellent catalyst for hydrogenation processes. ${ }^{52}$ The $\mathrm{Au} / \mathrm{TiC}(001)$ interactions produce a substantial polarization of electron density around the Au nanoparticle, which may facilitate the dissociation of $\mathrm{H}_{2}$, and make $\mathrm{H}$ atoms available.

In this way, Illas et al. ${ }^{53}$ point out that the $\mathrm{H}_{2}$ dissociation on the bare clean TMCs strongly depends on the chemical nature of the support, showing that the $\mathrm{H}_{2}$ molecule interacts rather strongly with $\mathrm{TiC}(001)$ and $\mathrm{ZrC}(001)$, but very weakly with $\mathrm{VC}(001)$ and $\delta-\mathrm{MoC}(001)$.

Here, we present a systematic study of the adsorption of ammonia-borane on several sites of the $\mathrm{TiC}(001)$ and $\mathrm{ZrC}(001)$ surfaces. The main objective of this study is to reveal possible trends in the interaction of the $A B$ molecule with different adsorption sites of the carbide surfaces, and to relate these tendencies with the possible activation of the supported molecule towards the dehydrogenation process. To the best of our knowledge, no previous theoretical work has been focused on studying the interaction of $A B$ on TMCs.

\section{Method of Calculation}

The adsorption of $A B$ on TMCs has been studied using periodic-boundary density functional theory calculations (DFT), carried out within the usual Kohn-Sham formalism, using a plane wave basis set with a cutoff of $450 \mathrm{eV}$ for the kinetic energy. The PBE (Perdew-Burke-Ernzerhof) ${ }^{55}$ form of the generalized-gradient approximation (GGA), has been chosen for the exchange-correlation potential, neglecting or including van der Waals interactions. In the case when van der Waals interactions are included, the method proposed by Grimme ${ }^{56}$ (D2) has been used. The effect of the atomic cores on the valence electron density has been considered by means of the projected-augmented plane wave (PAW). ${ }^{57}$ Calculations are performed using the Vienna ab initio Simulation Package VASP, version 5.2..$^{58}$ To carry out the necessary numerical integrations in the reciprocal space a Monkhorst-Pack grid $^{59}$ is used. A conjugated gradient algorithm with an energy criterion of $1 \times 10^{-5}$ $\mathrm{eV}$ is used for atomic position convergence, ensuring forces to be smaller than $8 \times 10^{-3} \mathrm{eV} / \AA$ in all cases. 
The surfaces are represented by slab models repeated periodically with a vacuum region of $20 \AA$. The slabs are constructed using the lattice parameter optimized for the bulk, which is $4.33 \AA$ for $\mathrm{TiC}(001)$ and $4.71 \AA$ for $\operatorname{ZrC}(001)$, with a $9 \times 9 \times 9$ k-point mesh. For each TMC, the most stable surface termination is used, which are the $\mathrm{TiC}(001)$ and $\mathrm{ZrC}(001)$ surfaces (Figure 1). Each final slab has four atomic layers, and corresponds to a $3 \times 3$ supercell (144 atoms) of the corresponding surfaces in the primitive cell. The dimensions of the simulation cells are $a=b=9.170 \AA$ and $c=28.646$ $\AA ̊$ for $\operatorname{TiC}(001)$, and $\mathrm{a}=\mathrm{b}=9.995 \AA$ and $\mathrm{c}=29.424 \AA$ for $\operatorname{ZrC}(001)$. In all cases the two outermost layers of one side of the slab are completely allowed to relax, whereas the two bottom layers are fixed at the bulk positions to provide an appropriate bulk environment for the surface layers. ${ }^{53,54,60,61}$ The $A B$ molecule on the TMCs surfaces $(A B / T M C(001))$ is simulated in all the cells described above $(144+6$ atoms) but the Kohn-Sham eigenfunctions are solved only in the $\ulcorner$ point of the supercell.

Once the final geometries for the stationary points in the $A B / T i C(001)$ and $A B / Z r C(001)$ systems are obtained, a vibrational analysis is carried out to characterize them either as minimum over the potential energy surface (PES), or as transition state structures (saddle points).

The adsorption energy $\left(E_{a d s}\right)$ between the $\mathrm{AB}$ molecule and the $\mathrm{TMC}(001)$ surfaces was evaluated as follows:

$$
E_{a d s}=\left\{E_{A B(a d s)}-\left(E_{A B}+E_{T M C(001)}\right)\right\}
$$

where $E_{A B(a d s)}$ is the total energy of the (001) surface with the adsorbed AB molecule, $E_{A B}$ is the total energy of the $\mathrm{AB}$ molecule in the gas phase, and $E_{T M C(001)}$ is the total energy of the relaxed TMC(001) surface.

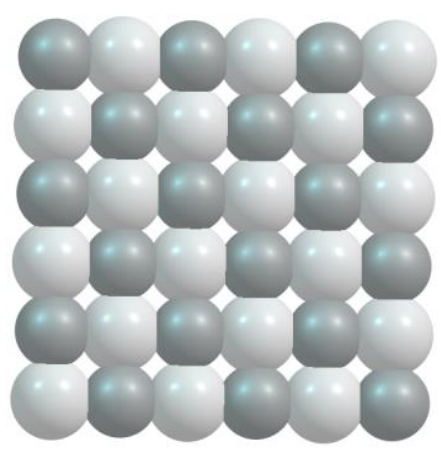

Figure 1. Top view of the $T M C(001)$ system. Transition metals (Ti or $\mathrm{Zr}$ ) are depicted in light gray hue while $\mathrm{C}$ in dark gray 
Table 1. Adsorption modes of $A B$ molecule on TiC (001), accounting for various ammonia-borane orientations

$$
\text { Bridge (b) Hollow (h) topC topTi }
$$

\section{Group 1}

\section{Configuration 1}

$\begin{array}{lllll}\text { A } & \mathrm{Bb} \perp & \mathrm{Bh} \perp & \mathrm{BtopC} \perp & \text { BtopTi } \perp \\ \mathrm{B} & \mathrm{Nb} \perp & \mathrm{Nh} \perp & \mathrm{NtopC} \perp & \mathrm{NtopTi}\end{array}$

\section{Group 2}

Configuration 2

\begin{tabular}{|c|c|c|c|c|}
\hline c & $\mathrm{Bb}-\mathrm{TiC}_{/ /}$ & $\mathrm{Bh}^{-\mathrm{TiC}_{/ /}}$ & BtopC-TiC $/ /$ & BtopTi-TiC $/ /$ \\
\hline R & $\mathrm{Nb}-\mathrm{TiC}_{/ /}$ & $\mathrm{Nh}-\mathrm{TiC}_{/ /}$ & NtopC-TiC $/ /$ & NtopTi-TiC $/ /$ \\
\hline & $\mathrm{BHb}^{-\mathrm{TiC}_{1 /}}$ & $\mathrm{BHh}^{-\mathrm{TiC}_{1 /}}$ & BHtopC-TiC $/ /$ & BHtopTi-TiC // \\
\hline & $\mathrm{NHb}-\mathrm{TiC}_{/ /}$ & $\mathrm{NHh}-\mathrm{TiC}_{/ /}$ & NHtopC-TiC $/ /$ & NHtopTi-Ti \\
\hline
\end{tabular}

\section{Configuration 3}

\begin{tabular}{|c|c|c|}
\hline G & $\mathrm{Bh}-\mathrm{CC}_{/ /}$ & BtopC-CC $/ /$ \\
\hline $\mathrm{H}$ & $\mathrm{Nh}-\mathrm{CC}_{/ /}$ & NtopC-CC $/ /$ \\
\hline 1 & $\mathrm{BHh}-\mathrm{CC}_{/ /}$ & BHtopC-C \\
\hline J & $\mathrm{NHh}-\mathrm{CC}_{/ /}$ & $\mathrm{NHtopC}-\mathrm{CC}_{/}$ \\
\hline
\end{tabular}

\section{Configuration 4}

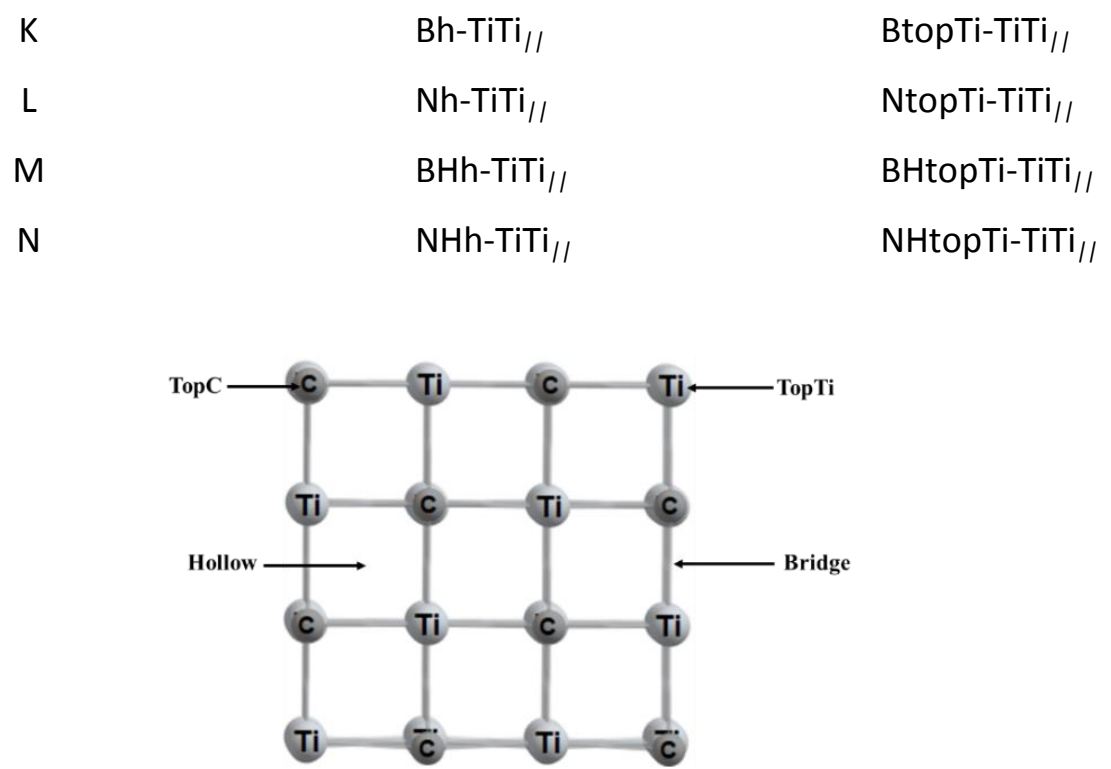

Figure 2. initial adsorption sites for the $\mathrm{BH}_{3} \cdot \mathrm{NH}_{3}$ on TiC (001) surface. 
Bridge (b) Hollow (h) topC topTi

\section{Group 1}

\section{Configuration1}

B)

$\mathrm{Nb} \perp$

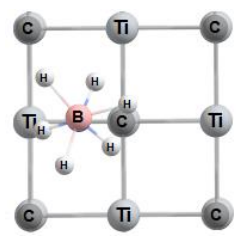

$\mathrm{Nh} \perp$

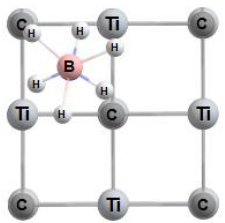

$\mathrm{NtopC}_{\perp}$

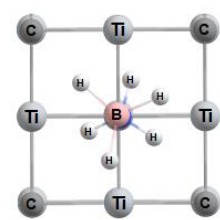

NtopTi

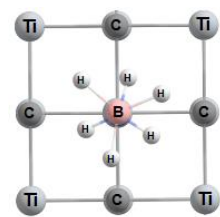

\section{Group 2}

\section{Configuration2}

D)

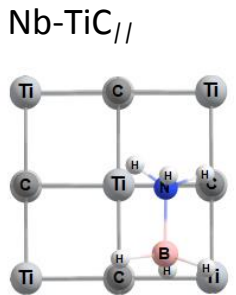

$\mathrm{Nh}_{\mathrm{TiC}}$

NtopC-TiC /

NtopTi-TiC $/ /$

F)
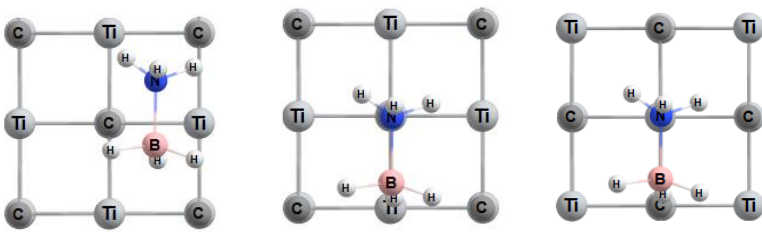

$\mathrm{NHb}-\mathrm{TiC}_{/ /}$

NHh-TiC $/$

NHtopC-TiC //

NHtopTi-TiC //
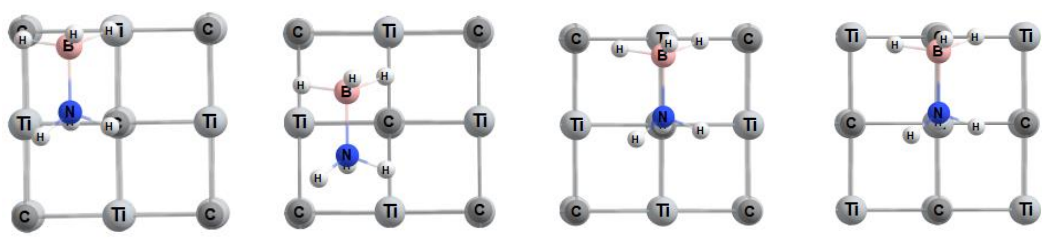

\section{Configuration3}

$\mathrm{H}$ y J)

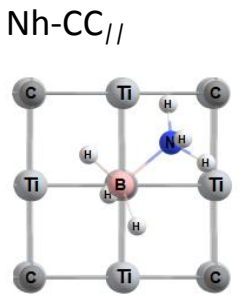

NtopC-CC //

$\mathrm{NHh}-\mathrm{CC}_{/ /}$

NHtopC-CC $/ /$
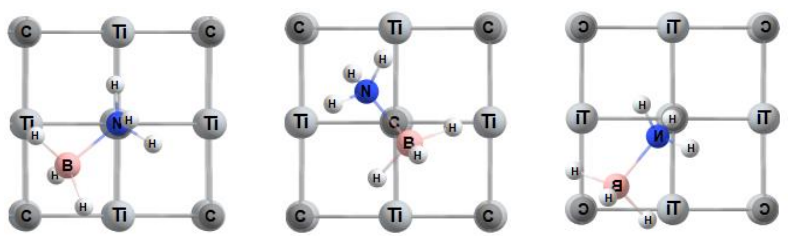

\section{Configuration4}

L y N)

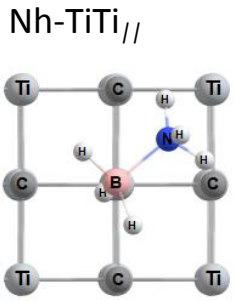

NHh-TiTi//

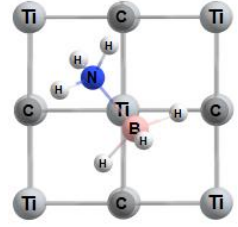

NHtopTi-TiTi//
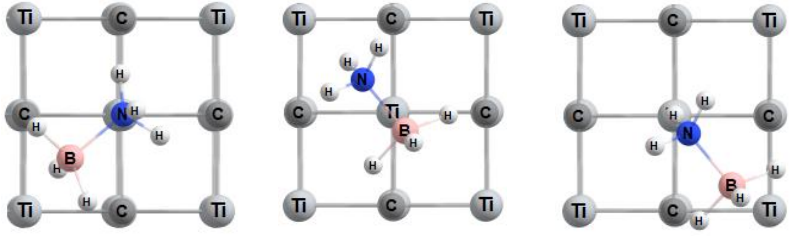

Figure 3. Adsorption modes of the $A B$ molecule on $\mathrm{TiC}(001)$, under configurations from the $\mathrm{N}$ atom. (The same modes were also considered from the $\mathrm{B}$ atom, see Figure $\mathrm{S} 1$ in $\mathrm{SI}$ ). 


\section{Results and discussion}

\subsection{Adsorption of AB molecule on $\mathrm{TiC}(001)$.}

We have investigated the adsorption of the $A B$ molecule on the $T i C(001)$ surface model, starting from different plausible adsorption sites denoted as: top ( $\mathrm{C}$ or $\mathrm{Ti}$ ), Bridge, and Hollow (standard four-fold hollow), as indicated in Figure 2. A large variety of orientations of $A B(40)$ with respect to $\mathrm{TiC}(001)$ was considered, as shown in Figure 3. Orientations are selected according to the interaction of the $A B$ molecule with the surface. These 40 orientations were divided into two principal groups and four configurations (see Table 1 and Figure 3). The groups are classified according to the orientation of the B-N bond relative to the surface. In Group 1, the B-N bond is perpendicular to the surface, while group 2 includes conformations when the B-N bond is parallel to the surface. Configuration 1 (group 1) is subdivided into two sets, depending on which atom is closer to the surface, boron (configuration A) or nitrogen (configuration B). Group 2 includes configurations 2, 3 and 4; each one is subdivided in four sets. In Configuration 2, the B-N axis is parallel to the $\mathrm{Ti}-\mathrm{C}$ direction of the surface. $\ln \mathrm{C}$ ) and $\mathrm{D}$ ) boron and nitrogen atoms are adsorbed on sites described above. In E) the closest atom to the surface is one of the hydrogens bound to boron, while in D) it is one of the hydrogens bound to nitrogen. In Configuration 3, the B-N axis is parallel to the $\mathrm{C}-\mathrm{C}$ direction of the surface with $\mathrm{G}), \mathrm{H}$ ), I) and J) in the same positions as in C), D), E) and F), but only on Hollow and topC sites. Finally, in Configuration 4, the B-N axis is parallel to the Ti-Ti direction of the surface with $\mathrm{K}$ ), L), M) and N) in the same positions as in $\mathrm{C}$ ), D), E) and F), but only on Hollow and topTi adsorption sites. Note that, in subgroups G) to N) only two adsorption sites are reported in Table 1, because all other possibilities are already included in subgroups A) to F).

\subsection{Effect of van der Waals on the adsorption of AB on TiC (001)}

The calculated adsorption energies with and without Van der Waals corrections for the 40 adsorption modes are shown in the Supporting Information (SI, Table S1). They were sorted in ascending order of energy, and the structures were categorized depending on whether they correspond to a product where a hydrogen atom has been released from the AB molecule (PSn, product-type structure), or whether they are just simple adsorption structures (ASn, adsorptiontype structure). Here, $n$ denotes a ranking of energetic stability ( $n=1$ is more stable than $n=2$ and so on; see Table S2 and S3 in SI). The structures were classified not only by energy but also by their 
structural parameters, because some adsorption structures, which correspond to chemisorptions, resulted in isoenergetic structures.

After structural relaxation obtained by using DFT without vdW corrections, the results indicate that the 40 initial configurations converged to only four different minima, labeled as PS1, PS2, AS1, and AS2, following a decreasing order of stability (binding energy). Most of the initial structures converged to AS1 (31), followed by AS2 (6), PS2 (2), and PS1 (1). Adsorption motifs AS1 and AS2 correspond to chemisorptions, while PS1 and PS2 correspond to a dehydrogenation of AB from boron with the dissociated $\mathrm{H}$ closer to the $\mathrm{C}$ than the Ti on the surface (see Figure 4a). The vdW corrections seem to have no effect on the diversity of adsorption structures, because the same minima (PS1, PS2, AS1, and AS2) are found even when vdW corrections are included in the structural optimization. The only difference is that a few initial structures do not converge to the same minimum they do with plain DFT. For instance, $73 \%$ of the initial structures converge to AS1 with vdWC, while $78 \%$ does so without vdW corrections.

Although the vdW correction does not always have a large effect on the structure or nature of the interaction between $A B$ and $T i C$, it does have a tremendous effect on the energetics of the interactions. Not including vdW corrections yields to an underestimation of about $50 \%$ of the interaction energy, as it can be seen in Table 2 and Table S1 in SI. Interestingly, for the AB molecule adsorbed on SiCNTs (Silicon Carbide Nanotubes), the structures that correspond to chemisorption (AS1 and AS2) are about $0.07 \mathrm{eV}$ more stable than those reported by Cao et al.. ${ }^{62}$

Table 2. Adsorption energies in eV, (Eads-1) and (Eads-3) with vdWC and (Eads-2) without vdW contribution, respectively.

\begin{tabular}{|l|c|c|c|}
\cline { 2 - 4 } \multicolumn{1}{c|}{} & \multicolumn{2}{c|}{ TiC } & ZrC \\
\hline Structure & $\mathbf{E}_{\text {ads-1 }}$ & $\mathbf{E}_{\text {ads-2 }}$ & $\mathbf{E}_{\text {ads-3 }}$ \\
\hline PS1 & $-2,28$ & $-1,44$ & -2.67 \\
PS2 & $-1,61$ & $-1,01$ & -1.52 \\
AS1 & $-0,86$ & $-0,42$ & -0.92 \\
AS2 & $-0,86$ & 0,39 & -0.99 \\
\hline
\end{tabular}




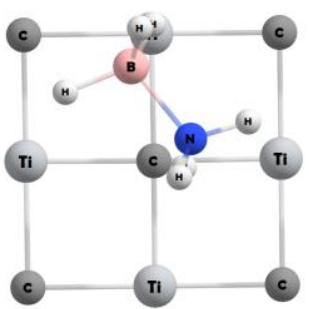

AS1

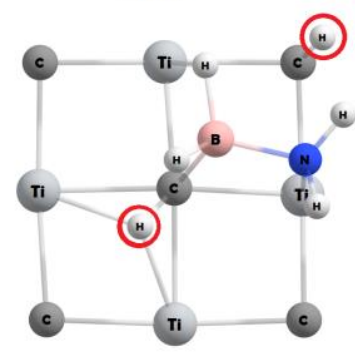

PS1

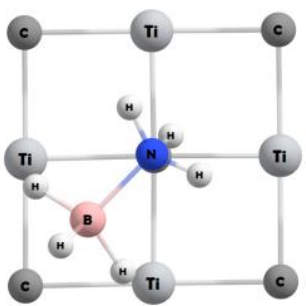

AS2

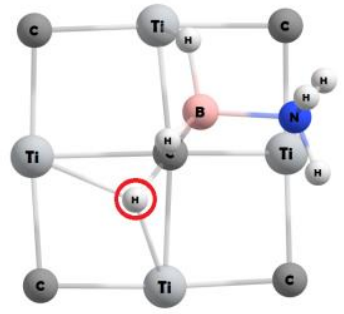

PS2

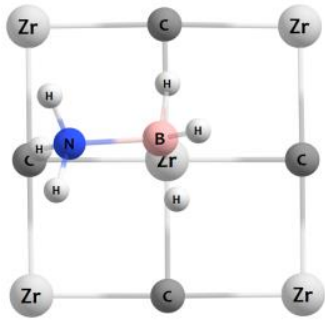

AS1

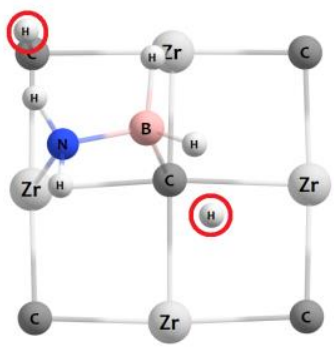

PS1

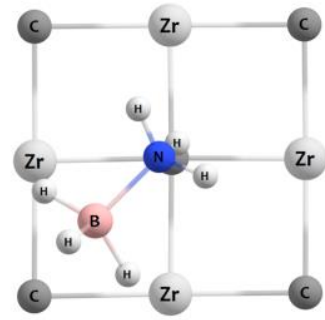

AS2

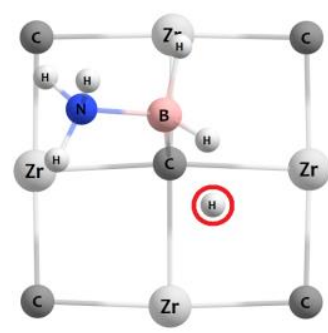

PS2

a)

b)

Figure 4. Modes of adsorption of the $A B$ molecule on the surface a) $\operatorname{TiC}(001)$ and b) $\operatorname{ZrC}(001)$, optimized with vdWC.

The adsorption of the $A B$ molecule on the $T i C(001)$ surface implies a closed-shell molecule with a large dipolar moment (5.1 D), and a surface with an appreciable degree of polarity. The interaction of the $A B$ molecule with the surface $T i C(001)$ results in a balance between the different intermolecular forces involved, such as: the attractive van der Waals dispersion forces, electrostatic, covalent bonding, and Pauli repulsion. Therefore, the influence of the long-range dispersion, neglected in the PBE exchange-correlation potential, can be crucial to describe the energetics of the interaction between the molecule and the surface. In most cases, the geometry of the $A B$ molecule and the surface were not modified with the inclusion of the long-range dispersion effects; however, when vdW corrections are included, structure PS1 shows a significant change of hybridization from $\mathrm{sp}^{2}$ to partial $\mathrm{sp}^{3}$, which implies that $\mathrm{NH}_{2} \mathrm{BH}_{2}$ adopts a tetrahedral geometry when adsorbed on the surface, contrary to the planar structure found without vdW corrections. Therefore, it is arguable that long-range dispersion effects should be included whenever possible, as in some cases it changes not only the energetics but also the nature of the adsorption. In this very case, plain DFT predicts a physisorption, while DFT+vdW predicts a chemisorption. The importance of using vdW correction has been reported in studies of adsorption of aromatic molecules on a surface of $\mathrm{Cu}(110),{ }^{63}$ thiophene on $\mathrm{Cu}(111),{ }^{64}$ adsorption $\mathrm{CO}_{2}$ and $\mathrm{CH}_{4}$ on $\delta$ - 
$\operatorname{MoC}(001),{ }^{65,66}$ Ethylene on $\delta-\operatorname{MoC}(001), \operatorname{TiC}(001)$ and $\mathrm{ZrC}(001),{ }^{67}$ and azobenzene on $\mathrm{TiO}_{2}(110) .{ }^{68}$ Consequently, all the posterior calculations in this research include vdW corrections.

\subsection{Adsorption of $\mathrm{AB}$ dimers on $\mathrm{TiC}(001)$}
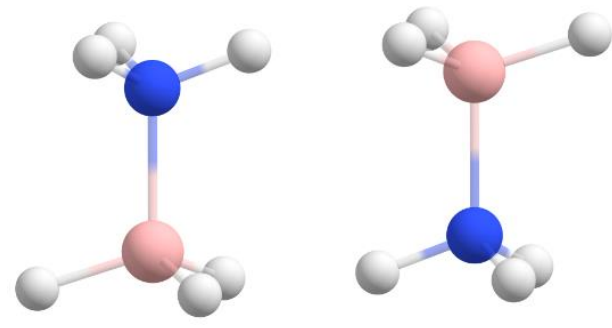

Figure 5: Geometry of $A B$ dimer.

To understand the role played by the interactions of dihydrogen $\mathrm{NH}-\mathrm{HB}$ in the process of dehydrogenation of $A B$ catalyzed by the TiC (001) support, we have adsorbed the $A B$ dimer (Figure 5 ) in this support. We did a gas-phase stochastic exploration using ASCEC (Simulated Annealing with Quantum Energy). ${ }^{69}$ The exploration of the PES for the $A B$ dimer consists of the production of candidate-structures, followed by the ASCEC stochastic procedure, and the optimizations of the feasible structures at the PW91/Def2-TZVP theory level. Only one minimum was obtained, the representative structure for the system, as shown in Figure 5. The initial positions of the $A B$ dimer were chosen to be consistent with the interactions observed in the most stable adsorption products (AS1, AS2). In addition, a motif perpendicular to the surface was also tested. Note that, in AS2 (Figure 4) boron remains in the hollow position, with the B-N bond parallel to the $\mathrm{C}-\mathrm{C}$ direction. However, this adsorption site is forbidden for the dimer (Figure 5), because it has a high sterical hindrance with other neighbor molecules.

Once the dimer sits on the surface, each molecule tends to place itself on the surface in the way a single molecule does (see Figure 6, D-AS1). That is, although the dipole-dipole interaction between molecules is still important, and it reduces the adsorption energy per $\mathrm{AB}$ molecule in about $0.2 \mathrm{eV}$ (compare Tables 3 and 2), it seems to have no effect on the mode of adsorption. Also, note for example, that in the perpendicular motif the dehydrogenation occurs from only one of the $A B$ fragments of the dimer. The differences between the bond lengths of the free $A B$ molecule and the $\mathrm{AB}$ dimer supported on TiC (001) are 0.03, 0.02 and $-0.05 \AA$ for $\mathrm{N}-\mathrm{H}, \mathrm{B}-\mathrm{H}$ and $\mathrm{B}-\mathrm{N}$, respectively. This shows that the $\mathrm{B}-\mathrm{N}$ bond is being shortened, and the $\mathrm{N}-\mathrm{H}$ and $\mathrm{B}-\mathrm{H}$ bonds are simultaneously lengthened. These values are similar to those obtained for the activation of the bonds when $A B$ is supported in TiC (see Table 4 of Section 3.5). Hence, although at a high coverage of the surface the dipole-dipole interaction weakens the chemisorption energy, it does not seem to change the way 
individual molecules adsorb on the TiC. Therefore, considering only the adsorption of one $A B$ molecule on the surface is a good approach to understand the adsorption process.

The values of the adsorption energies of the supported dimers, $A B-A B / T i C(001)$, as for the $A B / T i C(001)$ system, are consistent with chemisorption and dehydrogenated products (see Table 3).

Table 3. Adsorption energies per molecule in (eV) of dimer $\mathrm{AB}-\mathrm{AB} / \mathrm{TiC}(001)$

\begin{tabular}{|c|c|}
\hline Structure & $\mathbf{E}_{\text {ads }}$ \\
\hline AS1-like & -0.67 \\
\hline AS2-like & -0.67 \\
\hline Perpendicular & $-1,03$ \\
\hline
\end{tabular}

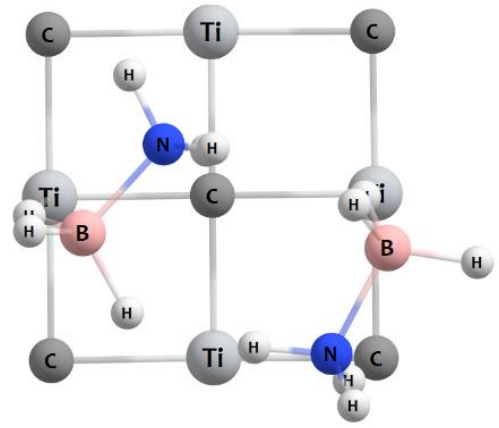

D-AS1

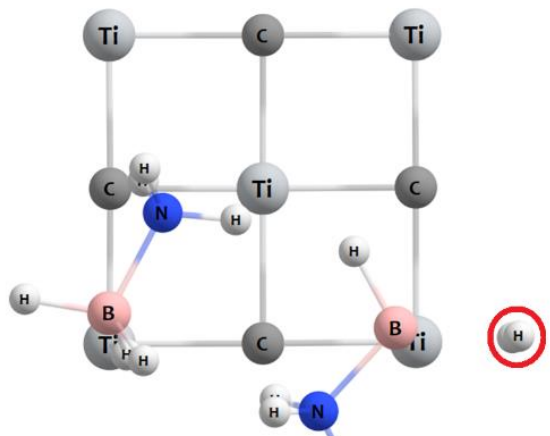

D-PS1

Figure 6. Structures resulting from the optimization of the geometries shown in Figure S3 in SI. The motifs AS1-like and AS2-like converge to D-AS1, and the perpendicular motif results in the D-PS1 geometry. The same nomenclature of ASn and PSn is preserved, but the letter D is prefixed, showing that the structure refers to a dimer.

\subsection{Hydrogen release from AB supported on TiC (001)}

From Figure 4a and Table 2 it is possible to observe that the structures PS1 and PS2 correspond to hydrogen-release products, which make them very stable (-2.28 and $-1.61 \mathrm{eV}$ of adsorption energy, respectively). Therefore, the support itself is capable not only of activating the bonds in the $A B$ molecule, but also of catalyzing the dehydrogenation process. In addition, this is consistent with the ability of a catalyst with localization of charge (as TiC) to release hydrogen from both, nitrogen and boron simultaneously, as it has been shown by some of the authors of this research ${ }^{70}$. Note that the first hydrogen is released from the $\mathrm{BH}_{3}$ fragment, which agrees with previously reported investigations. ${ }^{18,31,71,72}$ Those studies, both theoretical and experimental, attempted to elucidate the 
dehydrogenation mechanism of catalyzed $A B$ (either hydrolytically or thermally), showing that the first interaction between the catalyst and the $A B$ molecule happens through the hydrogen bonding to boron. Furthermore, the second hydrogen is released from the $\mathrm{NH}_{3}$ fragment. Here we find that for all the configurations evaluated, only one of them ends in the release of two hydrogens, one from each fragment of the molecule (see structure PS1 in Figure 4a).

The optimization of the configurations $\mathrm{Bb}_{/ /}, \mathrm{NtopTi} / /$, and $\mathrm{Nh}-\mathrm{CC}_{/ /}$, leads to geometries corresponding to the release of up to two hydrogen atoms from the $A B$ molecule (see Figure 7). This occurs when the boron and nitrogen atoms are placed at 2.2 and $2.6 \AA$ from the bridge, topTi, or hollow positions. Consequently, the hydrogen atom bonded to boron ends up at a distance of 1.42, 1.44 and $1.08 \AA$ to the closest carbon for the mentioned configurations, respectively. Our results reflect a trend between the $\mathrm{BH}-\mathrm{C}$ distance and the minima found in the PES, because a small $\mathrm{BH}-\mathrm{C}$ distance is particularly common to the three structures of dehydrogenated products. The breaking of the $\mathrm{H}-\mathrm{H}$ bond and the diffusion of the activated hydrogens on the $\mathrm{TiC}(001)$ surface has been explored before, ${ }^{52}$ showing that the activated hydrogen in the $\mathrm{TiC}(001)$ prefers to position itself over carbon atoms. Therefore, when the initial distance $\mathrm{BH}-\mathrm{C}$ is very small, the release of hydrogen is favored, and it tends to result in dehydrogenation.

The importance of the distance of $\mathrm{BH}$ to the surface becomes clear from the initial configurations $\mathrm{BHtopC}_{/ /}, \mathrm{BHtopC}-\mathrm{CC}_{/ /}, \mathrm{BHb}_{/ /}, \mathrm{NHb}_{/ /}, \mathrm{Nb}_{/ /}$, which do not release hydrogen but are geometrically similar to those in which dehydrogenation happens; the similarity being an interaction between the $\mathrm{BH}$ and $\mathrm{C}$ atoms. However, the main difference is in the distances between the $\mathrm{BH}$ bond and $\mathrm{C}$, which are greater than $2.2 \AA(2.23,2.49,2.44,2.46,2.22 \AA$, respectively), resulting in a physisorption or chemisorption. Accordingly, we checked whether the optimized geometry changes when the distance $\mathrm{BH}-\mathrm{C}$ in the $\mathrm{BH}$ topC // and $\mathrm{BH}$ topC- $\mathrm{CC}_{/ /}$structures is intentionally decreased, from 2.23 to $1.14 \AA$ and 2.49 to $1.17 \AA$, respectively. In this case, the structures converge to the previously reported minimum with the release of a hydrogen atom (PS2), revealing the existence of a barrier between the physical adsorption and the dehydrogenation. 


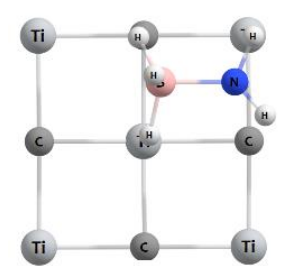

$\mathbf{B b}$

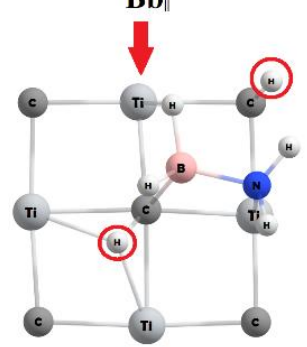

PS1

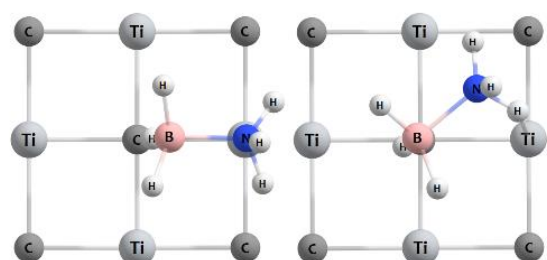

Nh-C $C_{\|}$

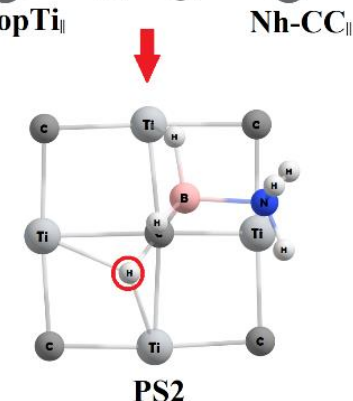

Figure 7. Adsorption geometries for the release of up to two hydrogen atoms when $A B$ is adsorbed on $\mathrm{TiC}(001)$ surface. The released hydrogens are marked with circles.

\subsection{Bond activation B-N, N-H, and B-H of AB molecule on TMC(001)}

The previous analyses indicate that the surface of TiC (001) does not act as a simple spectator, since, by itself, it can activate all the bonds in the $A B$ molecule and subsequently release hydrogen. On the other hand, previous studies on the dissociation of molecular hydrogen by Au that is supported on transition metal carbides, conclude that the most active system for $\mathrm{H}_{2}$ dissociation among all the metal carbides tested, is $\mathrm{Au}_{4} / \mathrm{ZrC}(001) .{ }^{53}$ Therefore, we have decided to evaluate the adsorption and possible release of $\mathrm{H}$ from $\mathrm{AB}$ using as support $\mathrm{ZrC}(001)$ in addition to $\mathrm{TiC}(001)$. When deciding on a starting point for studying the adsorption of $A B$ on $\operatorname{ZrC}(001)$, we take into account the initial exploration made in Section 3.1 for the adsorption of the AB molecule on TiC (001), and only consider the most stable structures obtained with vdW corrections (AS1 and AS2 in Figure 4a). The bond energies for the motifs studied are reported in Table 2.

Optimization of $\mathrm{ZrC}(001)$ yields four stable configurations, as shown in Figure 4b). Two of them are chemisorptions (AS1 and AS2), and the other two are dehydrogenation products. Unexpectedly, one of the chemisorptions (AS2) and one of the dehydrogenations (PS1) are structures very similar to AS1 and PS1 in TiC. Overall, the most stable chemisorption on $\operatorname{ZrC}$ is AS2, and the most stable dehydrogenation product is the double release in PS1 (see Table 2). 
Table 4. Variation in the bond distances (in A) B-N, N-H and B-H between the free AB molecule and supported on $\mathrm{TiC}(001)$ and $\mathrm{ZrC}(001)$. The number (2) in parentheses indicates that two bonds have been modified to this value. The negative sign indicates that the bond distance decreases when the $A B$ molecule is supported.

\begin{tabular}{|l|c|c|c|c|c|c|}
\hline \multirow{3}{*}{ Motives } & \multicolumn{3}{|c|}{$\begin{array}{l}\text { Differences in bond length (in A) of the } \\
\text { free AB molecule and supported on TiC }\end{array}$} & \multicolumn{3}{|c|}{$\begin{array}{c}\text { Differences in bond length (in A) of the } \\
\text { free AB molecule and supported on ZrC }\end{array}$} \\
\cline { 2 - 7 } & B-N & $\mathbf{N}-\mathbf{H}$ & B-H & B-N & $\mathbf{N}-\mathbf{H}$ & B-H \\
\hline AS1 & -0.04 & 0.02 & 0.03 & -0.04 & 0.04 & $0.01(2)$ \\
AS2 & -0.05 & 0.03 & $0.02(2)$ & -0.06 & 0.05 & $0.02(2)$ \\
\hline
\end{tabular}

In the optimized structure of the free $A B$ molecule, the length of the $B-N$ bond is $1.65 \AA$, $1.22 \AA$ for $B$ $\mathrm{H}$, and $1.02 \AA$ for $\mathrm{N}-\mathrm{H}$, which agree with other experimental and theoretical data. ${ }^{73,74}$ However, B-N, $\mathrm{N}-\mathrm{H}$, and $\mathrm{B}-\mathrm{H}$ bonds are activated when the $\mathrm{AB}$ molecule is adsorbed on $\mathrm{TMC}(001)$, as it can be seen in Table 4 . On average, for TiC and $\mathrm{ZrC}$ respectively, the B-H bond elongates by 0.02 and $0.01 \AA$, the $\mathrm{N}-\mathrm{H}$ one by 0.03 and $0.04 \AA$, and the $\mathrm{B}-\mathrm{N}$ bond shortens by 0.04 and $0.05 \AA$. . In the case of TiC(001), in both structures corresponding to chemisorption (AS1 and AS2), the B-N bond tends to shorten, while the $\mathrm{N}-\mathrm{H}$ and $\mathrm{B}-\mathrm{H}$ bonds tend to lengthen in almost equal proportion. Contrary, in $\mathrm{ZrC}(001)$ it seem easier to break the $\mathrm{N}-\mathrm{H}$ bond first. This implies that the $\mathrm{B}-\mathrm{N}$ bond is strengthened as the breaking of the $\mathrm{B}-\mathrm{H}$ and $\mathrm{N}-\mathrm{H}$ bonds is favored. Additionally, in the AS2 structure for both surfaces, the elongation of two B-H bonds is observed. From activation, it could be intuited that the release of the first hydrogen from the $A B$ molecule can result from both fragments in $\mathrm{TiC}$; whereas in $\mathrm{ZrC}$ the $\mathrm{N}-\mathrm{H}$ bond seems to break first. However, as it is observed in Figure 4a (PS2), and from all the adsorption sites explored, it is only possible to obtain the first hydrogen detached when this is released from the boron atom.

\subsection{Possible initial pathways of $A B$ dehydrogenation catalyzed by $T M C(001)$}

The possible pathways for the dehydrogenation of up to 2 hydrogen atoms from $A B$ are shown in scheme 1. 


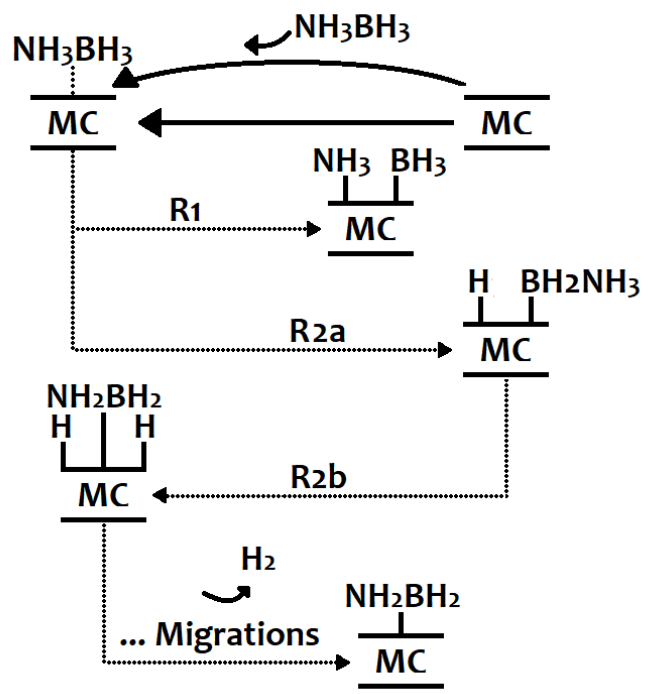

Scheme 1. Possible pathways for the dehydrogenation of $A B$, mediated with the supports $T M C, M=Z r, T i$.

The AS1 structure is taken as a starting point for the study of the possible hydrogen release products. Two pathways were found: the breaking of the B-N bond (denoted R1); and the dehydrogenation of the $A B$ molecule (R2) in consecutive steps, involving the release of 1 hydrogen atom from the $\mathrm{BH}_{3}$ fragment (R2a), followed by the release of the second hydrogen from the $\mathrm{NH}_{3}$ fragment $(\mathrm{R} 2 \mathrm{~b})$. Once the product of the $\mathrm{R} 2 \mathrm{~b}$ route $\left(\mathrm{NH}_{2} \mathrm{BH}_{2}+2 \mathrm{H}{ }^{*}\right)$ is obtained, the possible migrations of activated hydrogen on TMC are evaluated (Scheme 2). Subsequently, the possible dehydrogenation products for $\mathrm{BH}_{2} \mathrm{NH}_{2}$ on TMC are analyzed (Scheme 3).

Table 5. Adsorption energies (eV) for the route indicated in pathway 1 of Scheme 1 , which refers to the B-N bond break. $\mathrm{BH}_{3}$ and $\mathrm{NH}_{3}$ fragments are supported on $\mathrm{TMC}(001)$ with $\mathrm{M}=\mathrm{Ti}$ or $\mathrm{Zr}$. All possibilities for this bond breaking are shown in Scheme 1.

\begin{tabular}{|c|c|c|c|}
\hline $\begin{array}{c}\text { Initial } \\
\text { Position }\end{array}$ & $\begin{array}{c}\text { Final } \\
\text { Position }\end{array}$ & Eads $_{\text {TiC }}$ & $E_{\text {ads }}$ ZrC \\
\hline $\mathrm{H}_{3} \mathrm{~N}-\mathrm{C}, \mathrm{H}_{3} \mathrm{~B}-\mathrm{C}$ & $\mathrm{H}_{3} \mathrm{~N}-\mathrm{M}, \mathrm{H}_{3} \mathrm{~B}-\mathrm{C}$ & -1.97 & $-2,35$ \\
\hline $\mathrm{H}_{3} \mathrm{~N}-\mathrm{M}, \mathrm{H}_{3} \mathrm{~B}-\mathrm{M}$ & $\mathrm{H}_{3} \mathrm{~N}-\mathrm{M}, \mathrm{H}_{3} \mathrm{~B}-\mathrm{C}$ & $-2,01$ & $-2,35$ \\
\hline $\mathrm{H}_{3} \mathrm{~N}-\mathrm{C}, \mathrm{H}_{3} \mathrm{~B}-\mathrm{M}$ & $\mathrm{H}_{3} \mathrm{~N}-\mathrm{M}, \mathrm{H}_{3} \mathrm{~B}-\mathrm{C}$ & -1.98 & $-2,35$ \\
\hline $\mathrm{H}_{3} \mathrm{~N}-\mathrm{M}, \mathrm{H}_{3} \mathrm{~B}-\mathrm{C}$ & $\mathrm{H}_{3} \mathrm{~N}-\mathrm{M}, \mathrm{H}_{3} \mathrm{~B}-\mathrm{C}$ & -1.92 & $-2,32$ \\
\hline
\end{tabular}

For pathway 1, four different positions of adsorption of the $\mathrm{BH}_{3}$ and $\mathrm{NH}_{3}$ fragments are evaluated, where the initial and final positions, and the adsorption energy are shown in Table 5 (the final structures are shown in Figure S4 in SI). After trying different adsorptions modes of the fragments $\mathrm{BH}_{3}$ and $\mathrm{NH}_{3}$, it is found that the most thermodynamically favored configuration is with $\mathrm{N}$ placing 
itself on top of the metal, and B on top of the carbon. The same values of adsorption energy show that there is little interaction between adsorbed fragments. These products are more stable than any chemisorbed complex or dehydrogenation product. As an interesting result, the adsorption of the isolated fragments $\left(\mathrm{BH}_{3}\right.$ and $\left.\mathrm{NH}_{3}\right)$ is most exothermic with $\mathrm{ZrC}(001)$. However, it is important to recall that in all chemisorptions and dehydrogenation, the B-N bond is strengthened. Therefore, although the adsorption of free fragments is thermodynamically favored, it is expected that the energy that is needed to break the B-N bond is large enough to make the adsorption of free fragments much more unlikely than the release of 1 or 2 hydrogen atoms.

Pathway 2 is evaluated in the same way for both surfaces, TiC (001) and $\operatorname{ZrC~(001),~and~following~a~}$ two-step path: In the first step, (R2a), the adsorption structure AS1 is connected with the hydrogenrelease product (PS2). In the second step, (R2b), the PS2 state is connected with a state in which a second hydrogen is released from the nitrogen atom (PS1). The thermodynamic stability of the dehydrogenation products and the lowest adsorption geometry in energy does not differ significantly when using the $\operatorname{ZrC}(001)$ or $\mathrm{TiC}(001)$ surface, except for the instance when two hydrogens are released, during which the product difference between the two surfaces differs by around $0.39 \mathrm{eV}$.

\subsection{Diffusion of hydrogen on TMC (001)}

Once two hydrogens are released from the $A B$ to the TMC surface, the consequent hydrogen migration could have an impact on the stability of the products. We survey this by placing the hydrogens on different positions (see Scheme 2), and not necessarily in the atoms closest to the B and $\mathrm{N}$ of the $\mathrm{NH}_{2} \mathrm{BH}_{2}$, which are the sites where they place just after the dehydrogenation. Initial and optimized structures can be found in Figures S5, S6, and S7 of the SI. A total of 11 configurations were tried: the M1 pathway with the hydrogens over the metal atoms, and M3 with the hydrogens over the carbon atoms - each with 5 different initial positions, and M2 with one. Mxa refers to hydrogens close to $\mathrm{NH}_{2} \mathrm{BH}_{2}$. Mxb is the case in which one hydrogen is over the metal atom next to the $\mathrm{NH}_{2} \mathrm{BH}_{2}$, while the other is two metal atoms apart from the first one. In Mxc, the hydrogens are over two closest-neighbors metals, but far from $\mathrm{NH}_{2} \mathrm{BH}_{2}$. In $\mathrm{Mxd}$, the hydrogens are far from $\mathrm{NH}_{2} \mathrm{BH}_{2}$ and there is a metal atom separating them. Finally, in Mxe, the hydrogens are over the same atom, and $\mathrm{x}$ indicates whether it is pathway M1 or M3. 
Table 6 reports the energies of the migration products for both supports. For the M1a and M2 pathways, significant differences are seen in the products obtained when they are passing from one surface to the other, despite the fact that the initial geometries of the motifs are the same for both surfaces. When $\operatorname{ZrC}(001)$ is used, pathway M1a releases a third hydrogen atom, while in pathway M2 a stabilization of the hydrogen occurs on the metal atom when its immediate neighbor is another hydrogen located on a carbon (see Figure S6 and S7 in SI for M1a and M2, with TiC and $\mathrm{ZrC})$. For both supports there is a preference of the hydrogen for the carbon of the carbide, which agrees with other studies previously reported. ${ }^{52}$ In all cases, the adsorption energy decreases when the hydrogens are positioned on the same type of atom. However, it is observed that the hydrogen molecule, which tends to move away from the surface, is formed only if both hydrogens are on the same metal. Remarkably, in the cases where a hydrogen molecule is formed (Figures S6 and S7 in $\mathrm{SI})$, this is stabilized by its placement on top of the metal atom, which, for clean surfaces, is an unfavorable outcome. ${ }^{52}$ Moreover, it has been shown that, in the $A B$ dehydrogenation on the surface of SiCNTs, one of the $\mathrm{H}$ atoms is stabilized over $\mathrm{C}$, and the other over Si. ${ }^{62}$ In our case, hydrogen atoms are more stable on top of the $\mathrm{C}$ atom, independent of the type of support. Nevertheless, we tried to stabilize the hydrogen on the metal atom, but it was not possible, except for the case of the M2 pathway for $\mathrm{ZrC}$, where one of the hydrogens forms a bond with a neighboring carbon. In comparison with SiCNTs, on the supports used in our research, the metalhydrogen interaction seems to favor the hydrogen migration. When surfaces of SiCNTs are used, thermal and kinetically unfavorable processes are shown in the desorption, since the migration processes of activated hydrogens along the surface are highly endothermic, and the activation barriers for migration fluctuate around 1.26 and $1.99 \mathrm{eV} .{ }^{62}$ However, in the case of the $\mathrm{TiC}$ and $\mathrm{ZrC}$ supports, highly exothermic (up to $3 \mathrm{eV}$ ) processes occur, leaving thermal energy available for the migration and eventual release of hydrogen, which seems more likely when both hydrogens are found on the metal (see M1e in Figures $\mathrm{S} 6$ and $\mathrm{S7}$ of $\mathrm{SI}$ ). As this analysis is purely thermodynamic, in order to obtain conclusive results regarding the viability of the support, not only as a catalyst, but also as a hydrogen store, it becomes necessary to analyze the barriers of activation. 
Tabla 6. Adsorption energies (in eV) for the migration of two hydrogens using both supports

\begin{tabular}{|c|c|c|}
\hline $\begin{array}{c}\text { Initial } \\
\text { position }\end{array}$ & $\mathbf{M}=\mathbf{T i}$ & $\mathbf{M}=\mathbf{Z r}$ \\
\hline M1a & $-1,33$ & -2.89 \\
\hline M1b & $-3,08$ & $-3,53$ \\
\hline M1c & $-3,12$ & $-3,56$ \\
\hline M1d & $-3,08$ & $-3,53$ \\
\hline M1e & $-1,68$ & $-2,01$ \\
\hline M2 & $-3,08$ & $-1,51$ \\
\hline M3a & $-2,28$ & $-2,67$ \\
\hline M3b & $-3,15$ & $-3,61$ \\
\hline M3c & $-3,01$ & $-3,56$ \\
\hline M3d & $-3,08$ & $-3,58$ \\
\hline M3e & $-2,40$ & $-2,90$ \\
\hline
\end{tabular}

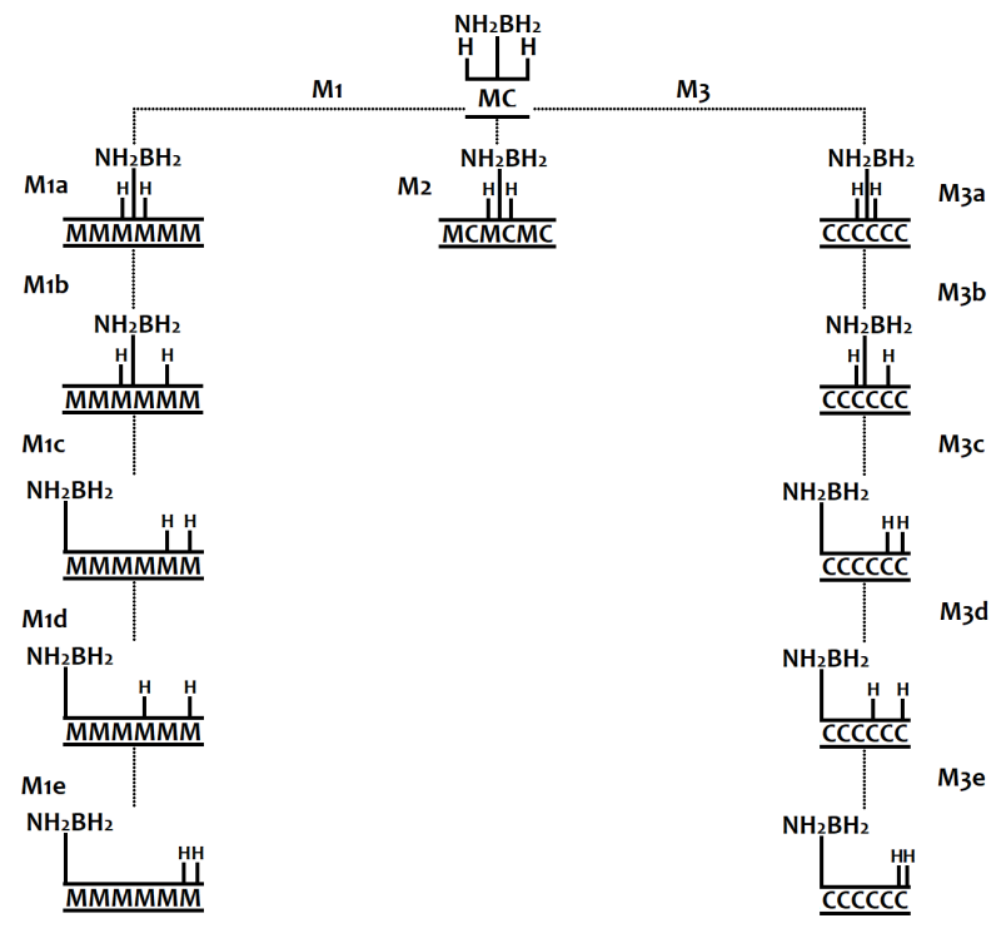

Scheme 2. Possible migrations for the two hydrogens released from $A B$ in both supports. "MMMMMM" and "CCCCCC" indicate that the hydrogens are in position along the diagonal of the metal ( $\mathrm{Zr}$ or Ti), or along the carbon diagonal. Additionally, "MCMCMC" refers to the position of the hydrogens along the row ordered as metal (Zr or Ti) -carbon 


\section{8. $\mathrm{NH}_{2} \mathrm{BH}_{2}$ dehydrogenation using $\mathrm{TMC}(001)$}

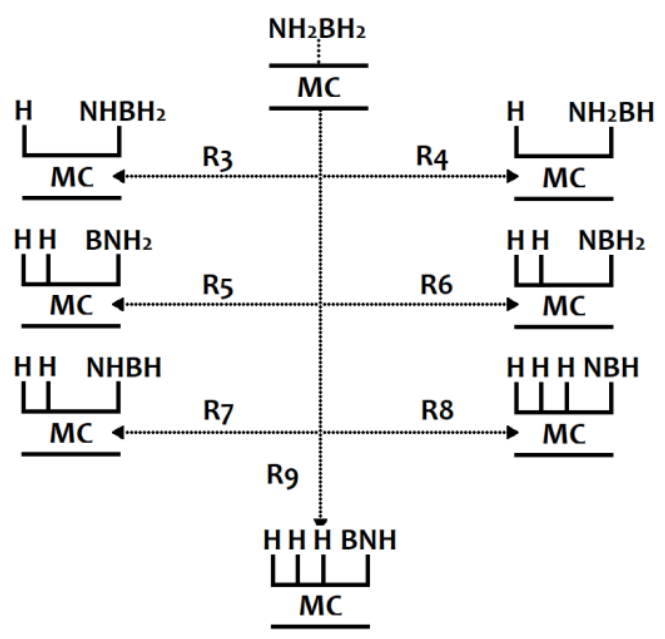

Scheme 3. Possible dehydrogenation pathways for $\mathrm{NH}_{2} \mathrm{BH}_{2}$ adsorbed on the $\mathrm{MC}, \mathrm{M}=\mathrm{Zr}$, and Ti (001) surfaces. The routes indicate from which fragment $\left(\mathrm{BH}_{3}\right.$ or $\left.\mathrm{NH}_{3}\right)$ the hydrogen is released.

To evaluate the capacity of the carbides in the dehydrogenation process of the $\mathrm{NH}_{2} \mathrm{BH}_{2}$ product, we have explored some adsorption sites. In this sense, Jiménez et al. ${ }^{67}$ studied acetylene activation using $\mathrm{TMC}(001)$, with $(\mathrm{M}=\mathrm{Ti}, \mathrm{Zr})$ as support. This molecule is isoelectronic to $\mathrm{NH}_{2} \mathrm{BH}_{2}$; therefore, exploration begins with the most stable adsorption site obtained by the authors, in addition to 4 more positions proposed by us.

For both supports, we include the configurations for the cases when the B-N bond is parallel to the surface. These configurations are defined according to the orientation of the B-N bond, described as follows: the B-N axis is parallel to TiC in A), to the $\mathrm{C}$ atoms of the surface in $\mathrm{B}$ ), and to Ti atoms of the surface in C), as shown in Figure S8, SI. In the case of configuration A), three geometries are studied: 1) B and $\mathrm{N}$ atoms at the bridge adsorption site. 2) The boron atom over the titanium atom and the nitrogen atom over the carbon atom of the surface. 3) Boron over carbon and nitrogen over titanium. It should be noted that, with respect to the earlier results obtained for the dehydrogenation of $A B$, we have ensured that in all cases the initial distance $B H-C$ is less than $1.5 \AA$. However, for both supports studied, only one adsorption mode with an energy of $-1.58 \mathrm{eV}$ for TiC, and $-1.92 \mathrm{eV}$ for $\mathrm{ZrC}$, is obtained (Figure 8). 


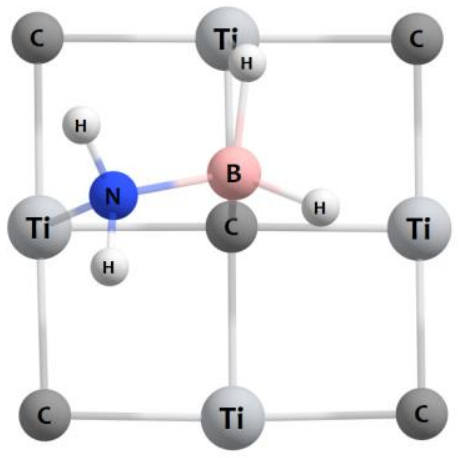

a) $\mathrm{NH}_{2} \mathrm{BH}_{2} / \mathrm{TiC}$

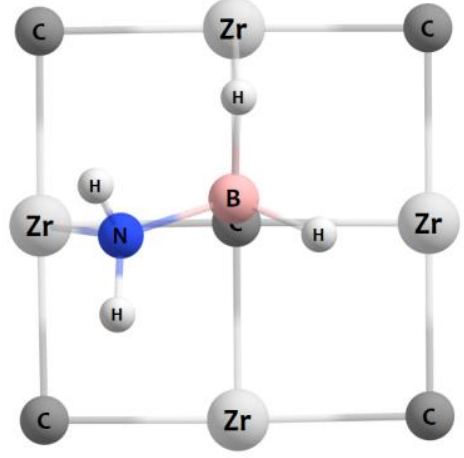

b) $\mathrm{NH}_{2} \mathrm{BH}_{2} / \mathrm{ZrC}$

Figure 8. Adsorption motif for $\mathrm{NH}_{2} \mathrm{BH}_{2}$ on the surfaces of a) $\mathrm{TiC}$ and b) $\mathrm{ZrC}$.

The results show that, even after the hydrogens are released from $A B$, the surface continues to activate and stabilize the dehydrogenation products (hydrogen atoms and $\mathrm{NH}_{2} \mathrm{BH}_{2}$ ). Table 7 reports the distances $\mathrm{B}-\mathrm{H}, \mathrm{N}-\mathrm{H}$ and $\mathrm{B}-\mathrm{N}$ for the $\mathrm{NH}_{2} \mathrm{BH}_{2}$ molecule, in its free and adsorbed on $\mathrm{TiC}$ and $\mathrm{ZrC}$ states. Note that, contrary to what happens with $A B$, the $B-N$ bond is stretched with respect to the free molecule $\mathrm{NH}_{2} \mathrm{BH}_{2}$. This is due to the loss of planarity caused by the interactions N-M and B-C. Activation at the $\mathrm{B}-\mathrm{H}$ bond is significant, with distances even larger than when the $A B$ molecule is adsorbed; hence, consecutive dehydrogenation is not ruled out. The ZrC surface manages to activate the molecule more than TiC.

Table 7. Variation in the bond distances (in $\AA$ ) $\mathrm{B}-\mathrm{N}, \mathrm{N}-\mathrm{H}$, and $\mathrm{B}-\mathrm{H}$, between the free $\mathrm{NH}_{2} \mathrm{BH}_{2}$ molecule and the one supported on $\mathrm{TiC}(001)$ and $\mathrm{ZrC}(001)$.

\begin{tabular}{|c|c|c|c|c|c|}
\hline \multirow{2}{*}{ Bond } & \multicolumn{2}{|c|}{$\begin{array}{c}\text { Bond distance for free } \mathrm{NH}_{2} \mathrm{BH}_{2} \text { molecule and } \\
\mathrm{NH}_{2} \mathrm{BH}_{2} \text { supported on } \mathrm{TiC} \text { and } \mathrm{ZrC}\end{array}$} & \multicolumn{2}{|c|}{$\begin{array}{c}\text { Variation in the bond } \\
\text { distance for } \mathrm{NH}_{2} \mathrm{BH}_{2} \\
\text { supported on } \mathrm{TiC} \text { and } \mathrm{ZrC}\end{array}$} \\
\cline { 2 - 6 } & $\mathbf{N H}_{2} \mathbf{B H}_{2}$ & $\mathbf{N H}_{2} \mathbf{B H}_{2} / \mathrm{TiC}$ & $\mathbf{N H}_{2} \mathbf{B H}_{2} / \mathbf{Z r C}$ & $\begin{array}{c}\text { Variation } \\
\text { TiC }\end{array}$ & $\begin{array}{c}\text { Variation } \\
\mathbf{Z r C}\end{array}$ \\
\hline $\mathbf{N}-\mathbf{H}$ & 1.015 & 1.020 & 1.022 & 0.005 & 0.007 \\
\hline B-H & 1.023 & 1.273 & 1.278 & 0.070 & 0.075 \\
& & 1.238 & 1.267 & 0.035 & 0.064 \\
\hline B-N & 1.393 & 1.562 & 1.575 & 0.169 & 0.182 \\
\hline
\end{tabular}

The stable position for $A B$ is obtained when the $B-N$ bond is almost parallel to the $M-C$ bond; while the most stable position for ethylene is when the $\mathrm{C}-\mathrm{C}$ bond is parallel to the $\mathrm{C}-\mathrm{C}$ direction of the surface. In our case, the planarity of the molecule is lost, and given the polarization of the molecule, 
it is expected that the nucleophilic nitrogen interacts with the surface titanium (electrophilic), while the boron atom with the carbon one. Considering that, for the $A B / T M C$ system the dehydrogenation process begins when the distance $\mathrm{BH}-\mathrm{C}$ is small, we evaluate if the proximity between the $\mathrm{BH}-\mathrm{C}$ atoms, within $\mathrm{BH}_{2} \mathrm{NH}_{2} / \mathrm{TMC}$, leads to a dissociation product. However, this does not occur; instead we obtain the same adsorption state. Therefore, the same interaction force that dominates the dehydrogenation of $A B$ does not prove to be effective in continuing the dehydrogenation of the product $\mathrm{NH}_{2} \mathrm{BH}_{2}$.

We intentionally increased the distance of the $\mathrm{N}-\mathrm{H}$ and $\mathrm{B}-\mathrm{H}$ bonds in the $\mathrm{BH}_{2} \mathrm{NH}_{2}$ system to evaluate different adsorption sites on TMC(001). All the initial positions are illustrated in Scheme 3, where 7 possible pathways are shown for the breaking of the $\mathrm{B}-\mathrm{H}$ and $\mathrm{N}-\mathrm{H}$ bonds. For pathways $\mathrm{R} 3, \mathrm{R} 4, \mathrm{R} 8$ and R9, two candidate structures are obtained in each case. For R5 and R6 pathways there is an initial candidate structure, while pathway R7 presents four candidate structures. All the initial structures are found in Figure S9 of SI.

Table 8 shows the adsorption energies for each process illustrated in Scheme 3 for both supports. A strong dependence of dehydrogenation energies with the geometry is observed. The energy released in the process is large, with the capacity of deforming the surface. Also, note that, in TiC, if the hydrogen to be migrated is bonded, or initially close to a titanium atom, and there is no other migration that compensates it, there seems to be no stabilization, and the molecule releases an additional hydrogen atom (R3b, R4b, R6, R7b). Contrary, in ZrC, in all cases, the hydrogens released correspond to those that are freed when the $\mathrm{NH}$ or $\mathrm{BH}$ bond are forced to break. This happens because there is a stabilization of type $\mathrm{H}-\mathrm{Zr}$, greater than the existing $\mathrm{H}-\mathrm{Ti}$, which makes the $\mathrm{TiC}$ surface more active towards dehydrogenation. There is also a clear stabilization of the hydrogens in two sites, the topC and the one similar to a bridge, with the topC site being the best site, which is in agreement with what was previously discussed. In addition, when the B-N bond is directed towards the carbon atoms, an additional stabilization is achieved. Finally, more stable products are obtained when the number of broken $\mathrm{B}-\mathrm{H}$ bonds is greater than the number of broken $\mathrm{N}-\mathrm{H}$ bonds. In most cases, the lowest energy products are obtained with the $\mathrm{ZrC}$ surface. 
Tabla 8. Energies (in eV) for the dehydrogenation pathways proposed in scheme 3 for $\mathrm{NH}_{2} \mathrm{BH} / \mathrm{MC}(001)$

\begin{tabular}{|c|c|c|c|}
\hline \multicolumn{2}{|c|}{$\begin{array}{c}\text { Initial } \\
\text { position }\end{array}$} & $\begin{array}{c}\text { Energy for Surface } \\
\text { TiC }\end{array}$ & $\begin{array}{c}\text { Energy for Surface } \\
\text { ZrC }\end{array}$ \\
\hline \multirow{2}{*}{ R3 } & R3a & -0.82 & -0.96 \\
\cline { 2 - 4 } & R3b & -1.17 & -1.66 \\
\hline \multirow{2}{*}{ R4 } & R4a & -2.62 & -2.83 \\
\cline { 2 - 4 } & R4b & -2.01 & -2.83 \\
\hline \multicolumn{2}{|c|}{ R5 } & -2.07 & -2.36 \\
\hline \multicolumn{2}{|c|}{ R6 } & -0.28 & -1.01 \\
\hline \multirow{4}{*}{ R7 } & R7a & -2.16 & -1.63 \\
\cline { 2 - 4 } & R7b & -0.89 & -2.62 \\
\cline { 2 - 4 } & R7c & -1.87 & -1.63 \\
\cline { 2 - 4 } & R7d & -2.01 & -2.18 \\
\hline \multirow{2}{*}{ R8 } & R8a & -1.20 & -1.77 \\
\cline { 2 - 4 } & R8b & -2.04 & -2.44 \\
\hline \multirow{2}{*}{ R9 } & R9a & 0.33 & -2.86 \\
\cline { 2 - 4 } & R9b & -2.22 & -2.67 \\
\hline
\end{tabular}




\section{CONCLUSIONS}

Calculations based on periodic DFT calculations using slab models and the Perdew-Burke-Ernzerhof exchange-correlation functional were used to systematically study 40 adsorption modes, which cover all interaction sites on the TiC (001) surface. The adsorption energy was considered with and without contributions of dispersive forces, using the Grimme method for the latter. After the optimization of all the adsorption sites, four structures were obtained, two of which are chemisorption structures, and the other two correspond to hydrogen-release products. These structures were taken as a starting point to study the adsorption of $A B$ supported on $\operatorname{ZrC}(001)$, considering dispersive forces. The inclusion of the $\mathrm{vdW}$ contribution influences the values of the adsorption energies, with these values being almost double in most of the studied sites, and, in some cases, changing the order of stability of the adsorbed structures. In most of the adsorption modes, the geometric parameters were not modified, except for the PS1 structure that releases two hydrogens, one from each fragment of the $\mathrm{AB}$ molecule, to form the $\mathrm{NH}_{2} \mathrm{BH}_{2}$ structure. The newly formed $\mathrm{NH}_{2} \mathrm{BH}_{2}$ shows a significant change when vdW corrections are included, given that it adopts a tetrahedral geometry, which implies that $\mathrm{NH}_{2} \mathrm{BH}_{2}$ is adsorbed on the surface, contrary to what was observed without vdW corrections, where $\mathrm{NH}_{2} \mathrm{BH}_{2}$ is planar. Therefore, long-range dispersion effects not only increase the adsorption energy, but also qualitatively change the geometry. Interestingly, we observe that, although the interaction $\mathrm{BH}-\mathrm{C}$ seems to not be favorable, since the hydrogen bond to boron is of hydride character with a negative partial charge, and the carbon of the surface is also negatively charged, we find that decreasing the $\mathrm{BH}-\mathrm{C}$ distance results in dehydrogenation products which are thermodynamically stable. Additionally, in the adsorption of $A B$ over $M C(001)$, the supports are not simple spectators, but they can simultaneously activate the $\mathrm{N}-\mathrm{H}$ and $\mathrm{B}-\mathrm{H}$ bonds in the molecule. Because of the high degree of polarity of the surfaces, both TiC (001) and ZrC (001) are predicted to release hydrogen from ammonia-borane, allowing the release of up to two $\mathrm{H}$ atoms, leaving as product the $\mathrm{NH}_{2} \mathrm{BH}_{2}$ structure, which is isoelectronic with ethylene.

Furthermore, the study of activated $\mathrm{H}$ migration on the supports results in processes that are favored thermodynamically, where there are products that are stabilized even at $3 \mathrm{eV}$ below the activated reagents. Although a kinetic exploration of the processes is not included in the results, we can infer that there is enough energy for the hydrogens to migrate through the surface to achieve the formation of $\mathrm{H}_{2}$, which is formed when both activated $\mathrm{H}$ atoms are close to the metal atom of the support. On the other hand, from the consecutive dehydrogenation, that is, $A B$ to 
$\mathrm{NH}_{2} \mathrm{BH}_{2}$ and from $\mathrm{NH}_{2} \mathrm{BH}_{2}$ to other products, as shown in Scheme 3, we can infer that the starting geometry significantly influences the final products, affecting the stability of the products formed. However, from the thermodynamic perspective, all processes are exothermic, with the products freed from these processes retaining enough energy to deform the surface.

\section{ACKNOWLEDGMENTS}

T.G. and C.C. acknowledge financial support provided by the FONDECYT No.11150164 and 1181121 projects. C. Z. H. is grateful to Universidad de Antioquia, project 16239. C.C. also acknowledges Financiamiento Basal para Centros Científicos y Tecnológicos de Excelencia-FB0807. The authors thank CONICYT-Programa de Cooperación Internacional-REDES150042. 